\title{
含噻唑联哌啶烟酰胺类化合物的合成与生物活性研究
}

\author{
丁成荣潘亚运殷许谭成侠*
}

(浙江工业大学化学工程与材料学院 杭州 310032)

\begin{abstract}
摘要 噻唑联哌啶结构能够影响生物体内胆固醇类化合物的代谢活动, 是氟噻唑吡乙酮抑制病原菌的氧胆固醇结合蛋 白(OSBP)的关键药效基团. 为了寻找具有生物活性的新型含噻唑联哌啶结构的化合物，设计并合成了 14 个未文献报 道的新颖含噻唑联哌啶烟酰胺类化生物, 其结构经 ${ }^{1} \mathrm{H} N \mathrm{NM} 、{ }^{13} \mathrm{C} \mathrm{NMR}$ 和 HRMS 确证, 生测结果显示: 在 $100 \mu \mathrm{g} / \mathrm{mL}$ 浓度下，目标化合物普遍具有抑菌活性，其中 1 个化合物对小麦赤霉病的抑菌活性为 $60 \%$; 3 个化合物对黄瓜灰霉病的 抑菌活性为 60\%; 6 个化合物对苹果褐斑病的抑菌效果为 70\%; (4-(5-(2,3-二氯苯基)-4-甲基噻唑-2-基)哌啶-1-基)(5,6-二 氯吡啶-3-基)甲酮(6k)对马铃薯晚疫病的抑菌活性为 75\%.
\end{abstract}

关键词 结构; 噻唑联哌啶; 烟酰胺; 抑菌活性

\section{Synthesis and Biological Activity of Thiazolidine Piperidine Nicotinamide Compounds}

\author{
Ding, Chengrong Pan, Yayun Yin, Xu Tan, Chengxia* \\ (College of Chemical Engineering and Materials Science, Zhejiang University of Technology, Hangzhou 310032)
}

\begin{abstract}
The structure of thiazolidine piperidine could affect the metabolic activitives of cholesterol compounds in vivo, and it is the key pharmacophore of oxythiazolidine to inhibit the oxygen cholesterol-binding protein (OSBP) of pathogenic bacteria. 14 novel thiazolidine piperidine nicotinamide derivatives were designed and synthesized in search of new bioactive compounds containing thiazolidine piperidine structure. The structures of target compounds were characterized by ${ }^{1} \mathrm{H}$ NMR, ${ }^{13} \mathrm{C}$ NMR and HRMS spectra. The preliminary bioassay showed that the target compounds generally had antibacterial activities. At the concentration of $100 \mu \mathrm{g} / \mathrm{mL}$, the antibacterial activity of one compound against Fusarium graminearum was $60 \%$, the antibacterial activities of three compounds against Botrytis cinerea were $60 \%$, the bacteriostatical activities of six compounds aganist Diplocarpon mali were 70\%, and the antibacterial activity of (4-(5-(2,3-dichlorophenyl)-4-methylthiazol-2yl)piperidin-1-yl)(5,6-dichloropyridin-3-yl)methanone (6k) against Phytophthora infestans (Mont.) de Bary was 75\%. Therefore it was worth for further research about structural optimization.
\end{abstract}

Keywords structure; thiazolidine piperidine; nicotinamide; antibacterial activity

含噻唑联哌啶结构作为一类蛋白抑制剂的药效基 团在医药领域 ${ }^{[1 \sim 3]}$ 有着广泛的应用，其作为药效基团在 农药领域的首次报道源于杜邦公司开发的氟噻唑吡乙 酮 ${ }^{[4]}$ (oxathiapiprolin)的问世, 氟噻唑吡乙酮结构包含异 噁唑、噻唑、哌啶、吡唑四个杂环, 是首个含噻唑联哌 啶酰胺类杀菌剂, 具有低毒、高效等特点, 尤其对卵菌 纲类病原菌有特效, 且对用户、农业生产者和环境的安 全性高, 因此深受各国推崇. 其作用机制是抑制病原菌 的氧胆固醇结合蛋白(OSBP). 近年来, 一些新颖具有不 同程度的杀菌活性的含噻唑联哌啶结构先导化合物 ${ }^{[5 ~ 15]}$
被陆续研发出来(Figure 1), 如 Lamberth 等 ${ }^{[16]}$ 开发的化 合物 1 在 $6 \mu \mathrm{g} / \mathrm{mL}$ 浓度下对马铃薯和番茄晚疫病，葡萄 霜霉病防治效果 $100 \%$; Choi 课题组 ${ }^{[17]}$ 开发的化合物 2 同时具有杀菌和杀虫活性(蚊子幼虫) $\mathrm{LC}_{50}=0.513 ; \mathrm{Li}$ 课 题组 ${ }^{[18]}$ 开发的化合物 3 在 $50 \mu \mathrm{g} / \mathrm{mL}$ 水稻纹枯病菌的抑 菌效果为 $93.75 \%$.

烟酰胺结构能够有效提高新化合物的电子传递活 性和亲水性，在生测过程中往往发现化合物具有更好的 生物活性、内吸性或选择性, 故而烟酰胺结构一直是新 农药创制的热点. 迄今为止已有不少商品化的产品问世

* Corresponding author. E-mail: tanchengxia@zjut.edu.cn

Received December 14, 2018; revised February 18, 2019; published online March 8, 2019.

Project supported by the Collaborative Innovation Center of Zhejiang Province Green Pesticide.

浙江省绿色农药 2011 协同创新中心开放基金资助项目. 
(图 1), 如德国巴斯夫公司开发啶酰菌胺(boscalid) ${ }^{[19]}$, 几乎对所有类型的真菌病害都有活性且不易产生交互 抗性; 如日本曹达公司开发的杀菌剂 picarbutrazox ${ }^{[20]}$, 其结构独特, 作用机制新颖, 具有渗透活性, 对盘梗霜 霉属、腐霉属、霜霉属、假霜霉属和疫霉属卵菌纲病原 菌有效.<smiles>Cc1cc(C(F)(F)F)nn1CC(=O)N1CCC(c2nc(NC(=O)c3cccc(Cl)c3O)cs2)CC1</smiles><smiles>CC(C)NC(=O)c1csc(C2CCN(C(=O)c3cc(Br)nn3-c3ncccc3Cl)CC2)n1</smiles>

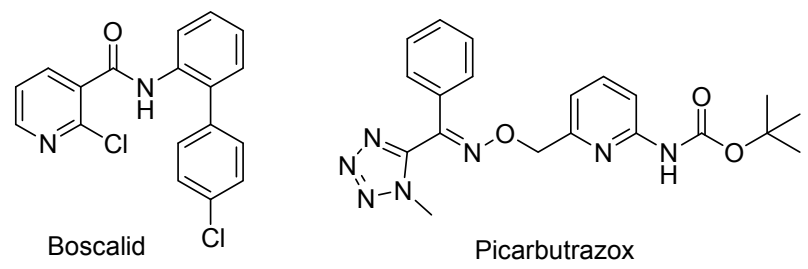

图 1 化合物 $1 \sim 3$ 和啶酰菌胺及 picarbutrazox 的结构式

Figure 1 Structures of compound $\mathbf{1} \sim \mathbf{3}$, boscalid and picarbutrazox

鉴于烟酰胺结构具有良好的生物活性, 研究者将烟 酰胺活性片段引入到含噻唑联哌啶结构中, 设计了含噻

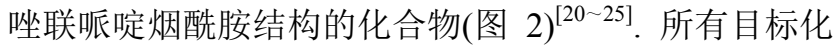
合物的结构经 ${ }^{1} \mathrm{H}$ NMR, ${ }^{13} \mathrm{C}$ NMR 和 HRMS 确认. 初步
生物活性测试表明：该类化合物具有良好的抑菌活性， 目标化合物的合成路线如图 3 所示.
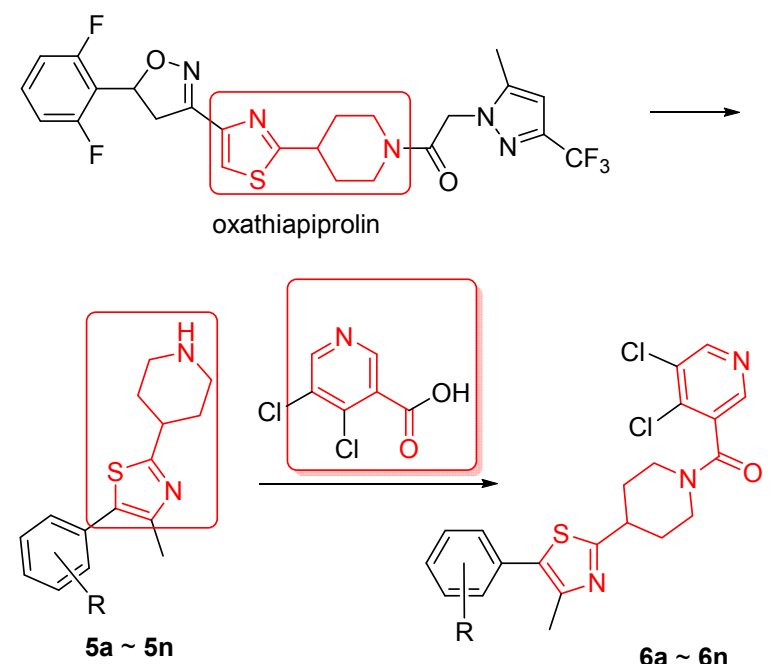

图 2 先导化合物的设计

Figure 2 Design strategy of lead compound

\section{1 结果与讨论}

\section{1 化合物的合成及结构分析}

以取代苯胺为底物, 经重氮化、氯化、环化得到中 间体 5, 中间体 5 脱保护后与 5,6-二氯烟酸反应最终得 到目标化合物 $6 \mathrm{a} \sim 6 \mathrm{n}$. 在合成过程中发现，中间体 2 的 合成是影响苯环上取代基多样性的主要原因，在其合成 过程中存在多取代和竞争反应，多取代情况可以通过控 制磺酰氯的用量和反应时间来抑制，竞争反应(图 4)则 无法控制，正常情况下 Chlorination 1 是反应的主要方 向，苯环上存在邻位取代基或强的供电子基团，能促进 反应往 Chlorination 2 的方向发展. 在苯环上邻位二取代 的情况下则以 Chlorination 2 的方向反应, 故而出现了

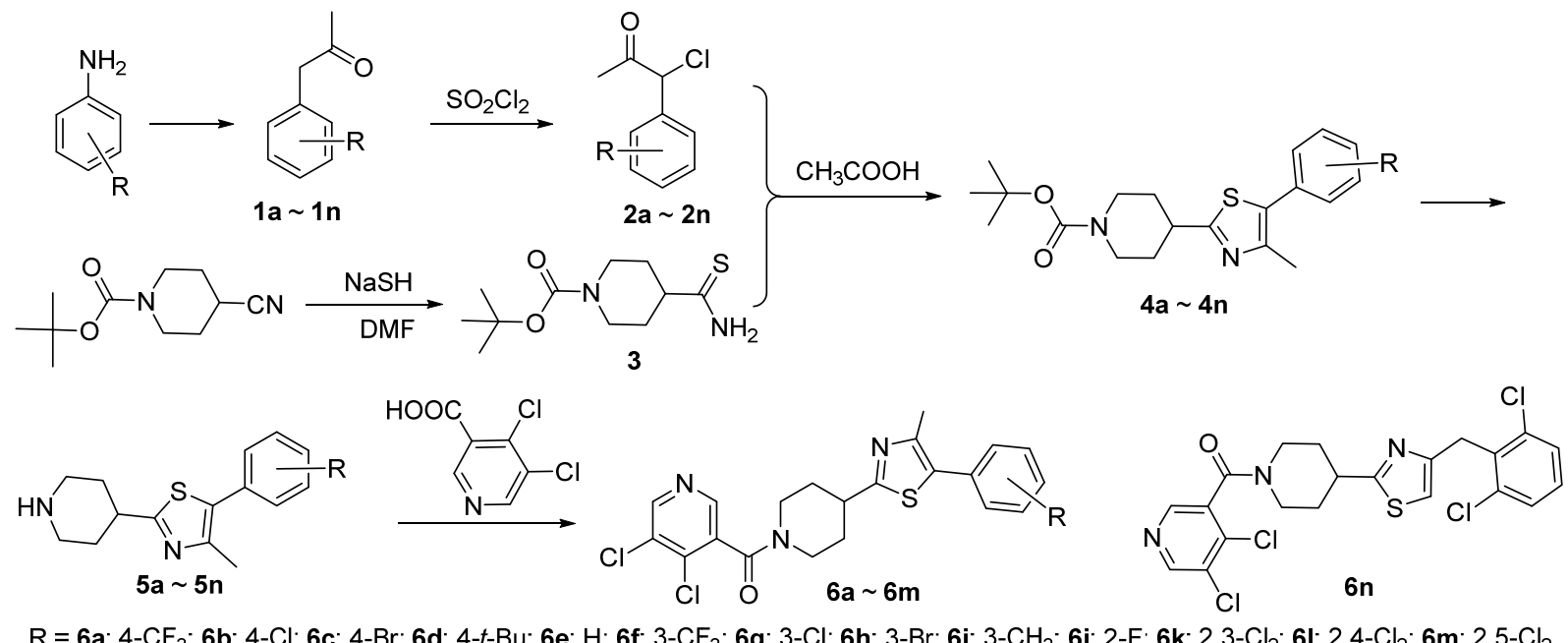

图 3 目标化合物的合成路线

Figure 3 Synthetic route of target compounds 
6n 的结构. 中间体 4 是关键中间体, 其噻唑环的合成利 用了 $\alpha$-卤代酮与硫代酰胺反应合成噻唑环的方式, 然而 在实验过程中发现, 在采用常规方法合成噻唑环, 即选 用 $N, N$-二甲基甲酰胺(DMF)、醇、丙酤等强极性液体为 溶剂, 碱为催化剂进行反应, 中间体 4 的含量低, 有大 量的反应底物未参与反应且不利于分离, 影响下一步反 应的进行. 在不断的研究和实践中发现, 中间体 2 与中 间体 3 的环合过程特殊, 环合过程需要在冰醋酸、无水 醋酸钠的条件下才能完全反应，同时反应温度控制在 $80{ }^{\circ} \mathrm{C}$, 温度过高会发生脱叔丁基羰基反应.

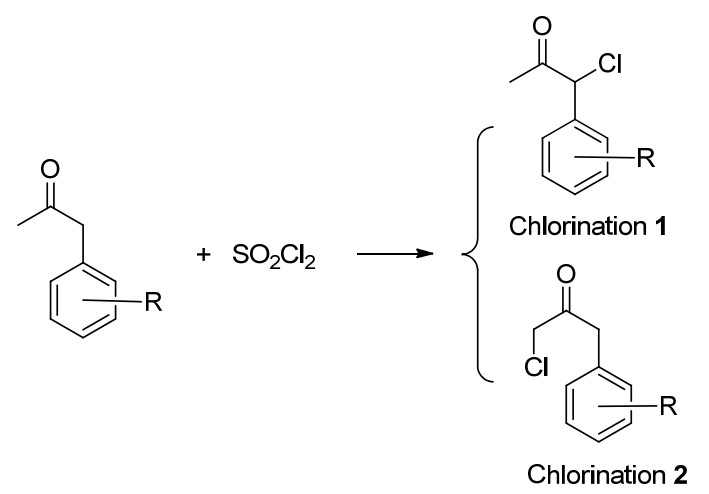

图 4 合成过程讨论

Figure 4 Discussion on the synthesis

以目标化合物 $6 \mathbf{a}$ 为例进行谱学分析, 分子式为 $\mathrm{C}_{22} \mathrm{H}_{18} \mathrm{Cl}_{2} \mathrm{~F}_{3} \mathrm{~N}_{3} \mathrm{OS},{ }^{1} \mathrm{H}$ NMR 谱图中 18 个氢均得到归属. $\delta$ $8.38(1 \mathrm{H}), 7.89(1 \mathrm{H})$ 为吡啶环的 $2 \mathrm{H} ; \delta 7.69(2 \mathrm{H})$, $7.54(2 \mathrm{H})$ 为苯环的 $4 \mathrm{H} ; \delta 2.51(3 \mathrm{H})$ 为噻唑环甲基的 $3 \mathrm{H} ; \delta$ $4.75(1 \mathrm{H}), 3.85(1 \mathrm{H}), 3.46 \sim 2.88(3 \mathrm{H}), 2.24(2 \mathrm{H}), 1.88$ $(2 \mathrm{H})$ 为哌啶环的 $9 \mathrm{H}$, 且哌啶环上的对称 $\mathrm{H}$ 往往会出现 偶合常数偏大的情况. ${ }^{13} \mathrm{C} N \mathrm{~N}$ 谱图中 22 个碳均得到归
属, 其中因苯环的对称关系存在重叠碳: $\delta 129.28(2 \times$ C), $125.63(2 \times \mathrm{C})$, 因三氟甲基中氟元素影响出现的碳 的裂峰 $\delta 129.70(\mathrm{q}, J=32.6 \mathrm{~Hz}), 125.63(\mathrm{q}, J=3.6 \mathrm{~Hz})$, 123.89 (q, $J=272.1 \mathrm{~Hz}$ ). 在 HRMS (ESI)谱图中该化合 物分子离子峰计算值为 $500.0572[\mathrm{M}+\mathrm{H}]^{+}$, 测定值 $500.0569[\mathrm{M}+\mathrm{H}]^{+}$, 绝对误差在 0.003 以内.

\section{2 化合物的抑菌活性}

目标化合物 $6 \mathbf{a} \sim \mathbf{6 n}$ 的抑菌活性数据列于表 1. 在 $100 \mu \mathrm{g} / \mathrm{mL}$ 浓度下，目标化合物对小麦赤霉病普遍具有 中等抑菌活性，其中 $\mathbf{6 j}$ 的抑菌活性达 $60 \%$. 4-氯苯基 $(6 \mathbf{b})$ 与 $2,4-$ 二氯苯基 $(61)$, 苯基 $(6 \mathrm{e})$ 与邻氟苯基 $(6 \mathbf{j}), 3$-氯 苯基 $(6 \mathbf{g})$ 与 2,3 -二氯苯基 $(6 \mathbf{k})$ 的比较发现, 苯环上的邻 位取代能够增强化合物对小麦赤霉病的抑菌活性; 目标 化合物对苹果褐斑病的抑制有特效，抑制效果普遍在 $50 \%$ 以上，其中 $6 \mathbf{a}, \mathbf{6 b}, \mathbf{6 e}, \mathbf{6 f}, \mathbf{6 i}, \mathbf{6 j}$ 的抑制效果达 $70 \%$. 苯基 $(6 \mathrm{e})$ 与取代苯基 $(6 \mathrm{a} \sim 6 \mathrm{~d}$ 和 $6 \mathrm{f} \sim 6 \mathrm{~m})$ 的比较发现, 苯 环上取代基的变化不是有效增强化合物对苹果褐斑病 抑菌活性的方式; 目标化合物对马铃薯晚疫病具有抑制 效果，其中 $6 \mathrm{k}$ 的抑制效果达 75\%. 苯基(6e)与单取代苯 基 $(6 \mathrm{a} \sim 6 \mathrm{~d}$ 和 $6 \mathbf{f} \sim 6 \mathbf{j})$ 和苯基 $(6 \mathrm{e})$ 与双取代苯基 $(6 \mathrm{k}, 6 \mathbf{6}$, $\mathbf{6 m})$ 的比较发现, 相邻的双取代可以作为增强马铃薯晚 疫病抑菌活性的方式; 目标化合物对黄瓜灰霉病具有中 等的抑制效果，其中 $6 \mathbf{b}, 6 \mathbf{e}, \mathbf{6 i}$ 的抑制效果达 $60 \%$. 与苹 果褐斑病的情况一致; $6 \mathbf{n}$ 杀菌活性的存在, 证实了在噻 唑环上双取代及另一侧单取代的可行性.

\section{2 结论}

将烟酰胺结构引入到含噻唑联哌啶类化合物, 设计 并合成了 14 个未见文献报道的新型含噻唑联哌啶烟酰

表 1 在 $100 \mu \mathrm{g} / \mathrm{mL}$ 浓度下目标化合物 $\mathbf{6 a} \sim \mathbf{6 n}$ 的抑菌活性(抑制率 $/ \%)^{a}$

Table 1 Antibacterial activity (inhibition/\%) of target compounds $6 \mathbf{a} \sim \mathbf{6 n}$ at $100 \mu \mathrm{g} / \mathrm{mL}$

\begin{tabular}{|c|c|c|c|c|c|c|}
\hline 化合物 & 取代基 & 小麦赤霉病 & 苹果褐斑病 & 水稻纹枯病 & 马铃薯晚疫病 & 黄瓜灰霉病 \\
\hline $6 a$ & $4-\mathrm{CF}_{3}$ & $50 \pm 4 \mathrm{bcd}$ & $70 \pm 5 b$ & 0 & $25 \pm 4 \mathrm{de}$ & $45 \pm 4 \mathrm{de}$ \\
\hline $6 \mathbf{b}$ & $4-\mathrm{Cl}$ & $30 \pm 3 \mathrm{e}$ & $70 \pm 8 b$ & 0 & $0 \pm 0 \mathrm{~h}$ & $60 \pm 3 b$ \\
\hline $6 c$ & $4-\mathrm{Br}$ & $45 \pm 5 \mathrm{~cd}$ & $65 \pm 6 b c$ & 0 & $15 \pm 3 \mathrm{fg}$ & $20 \pm 3 g$ \\
\hline 6d & $4-t-\mathrm{Bu}$ & $30 \pm 5 \mathrm{e}$ & $45 \pm 5 \mathrm{ef}$ & 0 & $0 \pm 0 \mathrm{~h}$ & $10 \pm 1 \mathrm{~h}$ \\
\hline $6 e$ & $\mathrm{H}$ & $50 \pm 6 b c d$ & $70 \pm 6 b$ & 0 & $40 \pm 5 c$ & $60 \pm 4 b$ \\
\hline $6 f$ & $3-\mathrm{CF}_{3}$ & $15 \pm 3 f$ & $70 \pm 7 b$ & 0 & $0 \pm 0 \mathrm{~h}$ & $30 \pm 3 f$ \\
\hline $6 \mathrm{~g}$ & $3-\mathrm{Cl}$ & $45 \pm 7 \mathrm{~cd}$ & $65 \pm 5 b c$ & 0 & $0 \pm 0 \mathrm{~h}$ & $45 \pm 3 \mathrm{de}$ \\
\hline $6 h$ & $3-\mathrm{Br}$ & $30 \pm 5 \mathrm{e}$ & $55 \pm 3 \mathrm{cde}$ & 0 & $10 \pm 1 \mathrm{~g}$ & $55 \pm 4 b c$ \\
\hline $6 \mathbf{i}$ & $3-\mathrm{CH}_{3}$ & $40 \pm 7 \mathrm{de}$ & $70 \pm 2 b$ & 0 & $0 \pm 0 \mathrm{~h}$ & $60 \pm 3 b$ \\
\hline $6 \mathbf{j}$ & $2-\mathrm{F}$ & $60 \pm 6 b$ & $70 \pm 4 b$ & 0 & $20 \pm 2 \mathrm{ef}$ & $50 \pm 4 \mathrm{~cd}$ \\
\hline $6 \mathbf{k}$ & $2,3-\mathrm{Cl}_{2}$ & $55 \pm 5 b c$ & $60 \pm 5 b c d$ & 0 & $75 \pm 6 b$ & $50 \pm 4 \mathrm{~cd}$ \\
\hline 61 & 2.4- $-\mathrm{Cl}_{2}$ & $50 \pm 10 \mathrm{bcd}$ & $55 \pm 7 \mathrm{cde}$ & 0 & $30 \pm 4 d$ & $50 \pm 5 \mathrm{~cd}$ \\
\hline $6 \mathrm{~m}$ & 2.5- $-\mathrm{Cl}_{2}$ & $55 \pm 4 b c$ & $50 \pm 3 \mathrm{def}$ & 0 & $10 \pm 1 \mathrm{~g}$ & $40 \pm 3 e$ \\
\hline $6 n$ & 2.6- $\mathrm{Cl}_{2}$ (异) & $15 \pm 2 \mathrm{f}$ & $40 \pm 4 f$ & 0 & $0 \pm 0 \mathrm{~h}$ & $40 \pm 3 e$ \\
\hline 多菌灵 & & $100 \pm 0 \mathrm{a}$ & $100 \pm 0 \mathrm{a}$ & $100 \pm 0$ & $100 \pm 0 \mathrm{a}$ & $100 \pm 0 \mathrm{a}$ \\
\hline
\end{tabular}

${ }^{a}$ 用 DPS 软件, Tukey 法多重比较, 小写字母表示差异达显著水平 $(P<0.05)$. 
胺类化合物, 其结构经 ${ }^{1} \mathrm{H} N \mathrm{NMR} 、{ }^{13} \mathrm{C} \mathrm{NMR}$ 和 HRMS 确 证. 生测结果显示: 在 $100 \mu \mathrm{g} / \mathrm{mL}$ 浓度下, 目标化合物 6j 对小麦赤霉病的抑菌活性为 $60 \% ; \mathbf{6 a}, \mathbf{6 b}, \mathbf{6 e}, \mathbf{6 f}, \mathbf{6 i}, \mathbf{6 j}$ 对苹果褐斑病的抑菌效果为 $70 \%$; 6k 对马铃薯晚疫病的 抑菌活性为 $75 \% ; 6 \mathbf{b}, \mathbf{6 e}, \mathbf{6 i}$ 对黄瓜灰霉病的抑菌活性为 $60 \%$. 所设计的含噻唑联哌啶烟酰胺结构是一种具有杀 菌活性的化合物, 对于开发一种新型的噻唑联哌啶烟酰 胺类杀菌剂具有很重要的研究意义和参考价值. 构效分 析显示：4-甲基-5-苯基-2-(哌啶-4-基)噻唑可以作为对烟 酸结构普篎的先导化合物, 进一步的结构优化和构效关 系仍在进行中.

\section{3 实验部分}

\section{1 仪器与试剂}

质谱采用 Bruker Daltonics Bio-TOF-Q III 型质谱仪 (ESIMS)测定; 天津光学仪器厂 Ry-1G 熔点仪, 温度计 未经校正. 核磁共振波谱使用 Bruker Avance 300 型. 核 磁共振仪测定. 所有试剂为国产市售分析纯.

\section{2 实验方法}

\subsubsection{1-取代苯基-2-丙酮 $(\mathbf{1 a} \sim \mathbf{1 n})$ 的合成}

在装有机械搅拌的 $250 \mathrm{~mL}$ 三口瓶中加入取代苯胺 (0.15 mol), 30\%浓硫酸 $(80 \mathrm{~mL})$. 在 $0{ }^{\circ} \mathrm{C}$ 条件下, 用恒压 滴液漏斗滴加 $\mathrm{NaNO}_{2}(0.15 \mathrm{~mol})$, 滴加完毕, 保温 30 $\min$, 在 $0{ }^{\circ} \mathrm{C}$ 条件下用恒压滴液漏斗滴加 $40 \% \mathrm{HBF}_{4}$ $(0.19 \mathrm{~mol})$, 保温 $3 \mathrm{~h}$, 减压抽滤, 得到氟硼酸重氮盐. 用 冰乙醇洗涤, 干燥除水. 在装有机械搅拌的 $250 \mathrm{~mL}$ 四 口瓶中加入醋酸钠 $(0.25 \mathrm{~mol}) 、 乙$ 酸异丙烯酯 $(0.80 \mathrm{~mol})$ 、 氧化亚铜 $(0.02 \mathrm{~mol})$, 将干燥的氟硼酸重氮盐分批次慢 慢滴加到四口瓶中, 控制温度在 $35 \sim 40{ }^{\circ} \mathrm{C}$ 反应 $8 \mathrm{~h}$. 抽 滤除盐, 滤饼用石油醚洗涤, 收集滤液, 减压蒸馏得到 中间体 $\mathbf{1 a} \sim 1 \mathbf{n}^{[26]}$, 淡黄色液体. 分析数据与参考文献报 道一致。

\subsubsection{1-取代苯基-2-氯-丙酮 $(\mathbf{2} \mathbf{a} \sim \mathbf{2 n})$ 的合成}

在 $50 \mathrm{~mL}$ 三口瓶中加入中间体 $1(0.05 \mathrm{~mol})$ 、二氯 甲烷 $(10 \mathrm{~mL})$, 采用磁力搅拌, 用恒压滴液漏斗缓慢滴加 磺酰氯 $(0.05 \sim 0.055 \mathrm{~mol})$, 反应 $2 \mathrm{~h}$. 用饱和碳酸氢钠溶 液调 $\mathrm{pH}=8 \sim 9$, 用二氯甲烷萃取, 无水硫酸钠干燥, 旋 蒸得中间体 $\mathbf{2} \mathbf{a} \sim \mathbf{2 n}^{[27]}$, 淡黄色液体, 直接投入下一步反 应.

\subsection{3 $N$-叔丁基羰基-4-硫代酰胺哌啶(3)的合成}

在带机械搅拌的 $250 \mathrm{~mL}$ 的四口烧瓶中加入 $70 \%$ $\mathrm{NaSH}(0.18 \mathrm{~mol}), \mathrm{NH}_{4} \mathrm{Cl}(0.18 \mathrm{~mol}), \mathrm{DMF}$ (120 mL), $N$ 叔丁基羰基-4-氭基哌啶 $(0.04 \mathrm{~mol})$, 在室温下搅拌 $72 \mathrm{~h}$. 反应完全后, 在摚拌过程中将反应液慢慢加入到 $600 \mathrm{~g}$
冰水中，搅拌 $1 \mathrm{~h}$ 后，出现大量白色固体. 抽滤，滤液用 乙酸乙酯萃取 $(100 \mathrm{~mL} \times 3)$, 旋蒸, 所得固体与滤饼合并 烘干得中间体 3, 白色固体，收率 95.3\%. m.p. 134 $135{ }^{\circ} \mathrm{C}$; ${ }^{1} \mathrm{H}$ NMR (500 MHz, Chloroform-d) $\delta: 7.66$ (s, $1 \mathrm{H}), 7.10$ (s, 1H), $4.22(\mathrm{~d}, J=12.5 \mathrm{~Hz}, 2 \mathrm{H}), 2.78 \sim 2.65$ (m, 3H), 1.90 (d, $J=12.6 \mathrm{~Hz}, 2 \mathrm{H}), 1.73$ (qd, $J=12.9,4.5$ $\mathrm{Hz}, 2 \mathrm{H}), 1.46$ (s, 9H); ${ }^{13} \mathrm{C}$ NMR (126 MHz, Chloroform-d) $\delta: 172.26,154.71,147.35,133.73,130.72,130.37(2 \times \mathrm{C})$, 129.64, $128.90(2 \times \mathrm{C}), 79.65,43.71,40.81(2 \times \mathrm{C}), 32.47$ $(2 \times \mathrm{C}), 28.477(3 \times \mathrm{C}), 16.04$. HRMS (ESI) calcd for $\mathrm{C}_{20} \mathrm{H}_{25} \mathrm{ClN}_{2} \mathrm{O}_{2} \mathrm{SNa}[\mathrm{M}+\mathrm{Na}]^{+}$415.1395, found 415.1403.

3.2.4 4-(4-取代苯基-5-甲基噻唑-2-基)哌啶-1-甲酸 叔丁酯 $(4 a \sim 4 n)$ 的合成

在 $100 \mathrm{~mL}$ 单口瓶中加入中间体 $2(0.05 \mathrm{~mol})$ 、中间 体 3 (0.05 mol)、无水醋酸钠 $(0.15 \mathrm{~mol})$ 、冰醋酸 $(50 \mathrm{~mL})$. 采用磁子搅拌, 在 $80{ }^{\circ} \mathrm{C}$ 温度下反应 $24 \mathrm{~h}$ 后, 旋蒸去除 冰醋酸. 往体系中加入乙酸乙酯, 过滤, 旋蒸得到褐色 的油状物，经柱分离后得到中间体 $\mathbf{4 a} \sim \mathbf{4 n}$, 白色固体. 直接用于下一步反应.

\subsubsection{4-取代苯基-5-甲基噻唑-2-基哌啶-1-盐酸盐} $(5 a \sim 5 n)$ 的合成

在 $100 \mathrm{~mL}$ 单口瓶中加入中间体 4 (0.05 mol)和 4 $\mathrm{mol} / \mathrm{L} \mathrm{HCl}$ 的二氧六环溶液 $(20 \mathrm{~mL})$. 在室温下采用磁子 搅拌，反应 $4 \mathrm{~h}$ 后旋蒸得到中间体 $\mathbf{5 a} \sim \mathbf{5 n}$, 固体或油状 物. 直接投入下一步反应.

\subsection{6目标化合物 $\mathbf{6 a} \sim \mathbf{6 n}$ 的合成}

在封管中加入中间体 5 (1.0 mmol)、 $N, N$-二异丙基 乙胺 $(5 \mathrm{mmol}) 、 5,6$-二氯烟酸 $(1.1 \mathrm{mmol}) 、 \mathrm{EDCl}(1.5$ $\mathrm{mmol}) 、 \mathrm{HOBt}(1.5 \mathrm{mmol}) 、$ 二氯甲烷 $(4 \mathrm{~mL})$. 反应 $24 \mathrm{~h}$ 后往体系中加入二氯甲烷 $(30 \mathrm{~mL})$, 多次水洗后, 旋蒸, 过柱得到目标化合物 $\mathbf{6 a} \sim \mathbf{6 n}$.

(5,6-二氯吡啶-3-基)(4-(4-甲基-5-(4-(三氟甲基)苯 基)噻唑-2-基)哌啶-1-基)甲酮(6a)：收率 $65.8 \%$ ，淡黄色 固体. m.p. $168 \sim 169{ }^{\circ} \mathrm{C} ;{ }^{1} \mathrm{H}$ NMR $(500 \mathrm{MHz}$, Chloroform- $d$ ) $\delta: 8.38(\mathrm{~d}, J=2.1 \mathrm{~Hz}, 1 \mathrm{H}), 7.89(\mathrm{~d}, J=2.1 \mathrm{~Hz}$, 1H), 7.69 (d, $J=8.4 \mathrm{~Hz}, 2 \mathrm{H}), 7.54$ (d, $J=8.4 \mathrm{~Hz}, 2 \mathrm{H}), 4.75$ $(\mathrm{s}, 1 \mathrm{H}), 3.85(\mathrm{~s}, 1 \mathrm{H}), 3.46 \sim 2.88(\mathrm{~m}, 3 \mathrm{H}), 2.51(\mathrm{~s}, 3 \mathrm{H})$, $2.24(\mathrm{~d}, J=17.6 \mathrm{~Hz}, 2 \mathrm{H}), 1.88(\mathrm{~d}, J=8.2 \mathrm{~Hz}, 2 \mathrm{H}) ;{ }^{13} \mathrm{C}$ NMR (126 MHz, Chloroform-d) $\delta: 171.22,165.20,150.21$, $148.26,145.12,137.61,135.65,131.60,130.84,129.70(\mathrm{q}$, $J=32.6 \mathrm{~Hz}), 129.60,129.28(2 \times \mathrm{C}), 125.63(\mathrm{q}, J=3.6 \mathrm{~Hz})$ $(2 \times \mathrm{C}), 123.89$ (q, $J=272.1 \mathrm{~Hz}), 47.51,42.09,40.18$, $32.75,31.77,16.06$. HRMS (ESI) calcd for $\mathrm{C}_{22} \mathrm{H}_{19} \mathrm{Cl}_{2} \mathrm{~F}_{3^{-}}$ $\mathrm{N}_{3} \mathrm{OS}[\mathrm{M}+\mathrm{H}]^{+}$500.0572, found 500.0569.

(4-(5-(4-氯苯基)-4-甲基噻唑-2-基)哌啶-1-基)(5,6- 
二氯吡啶-3-基)甲酮(6b): 收率 64.2\%, 白色固体. m.p. 172 $173{ }^{\circ} \mathrm{C} ;{ }^{1} \mathrm{H}$ NMR (500 MHz, Chloroform- $d$ ) $\delta: 8.38$ (d, $J=2.1 \mathrm{~Hz}, 1 \mathrm{H}), 7.89$ (d, $J=2.2 \mathrm{~Hz}, 1 \mathrm{H}), 7.45 \sim 7.38$ (m, 2H), $7.39 \sim 7.32(\mathrm{~m}, 2 \mathrm{H}), 4.75(\mathrm{~s}, 1 \mathrm{H}), 3.84(\mathrm{~s}, 1 \mathrm{H})$, 3.31 (tt, $J=11.2,3.9 \mathrm{~Hz}, 2 \mathrm{H}), 3.06(\mathrm{~s}, 1 \mathrm{H}), 2.47$ (s, 3H), $2.23(\mathrm{dd}, J=25.2,9.7 \mathrm{~Hz}, 2 \mathrm{H}), 1.87(\mathrm{~d}, J=47.4 \mathrm{~Hz}, 2 \mathrm{H})$; ${ }^{13} \mathrm{C}$ NMR (126 MHz, Chloroform- $d$ ) $\delta: 170.48,165.15$, $150.15,147.51,145.10,137.57,133.77,131.61,130.80$, $130.37,130.27(2 \times \mathrm{C}), 129.85,128.84(2 \times \mathrm{C}), 47.56$, 42.16, 40.14, 32.61, 31.89, 15.94. HRMS (ESI) calcd for $\mathrm{C}_{21} \mathrm{H}_{19} \mathrm{Cl}_{3} \mathrm{~N}_{3} \mathrm{OS}[\mathrm{M}+\mathrm{H}]^{+}$466.0309, found 466.0308.

(4-(5-(4-溴苯基)-4-甲基噻唑-2-基)哌啶-1-基)(5,6二氯吡啶-3-基)甲酮(6c): 收率 66.5\%, 白色固体. m.p. 177 $\sim 178{ }^{\circ} \mathrm{C}$; ${ }^{1} \mathrm{H}$ NMR (500 MHz, Chloroform- $d$ ) $\delta: 8.38$ (d, $J=2.1 \mathrm{~Hz}, 1 \mathrm{H}), 7.88(\mathrm{~d}, J=2.1 \mathrm{~Hz}, 1 \mathrm{H}), 7.63 \sim 7.50$ $(\mathrm{m}, 2 \mathrm{H}), 7.38 \sim 7.20(\mathrm{~m}, 2 \mathrm{H}), 4.74(\mathrm{~s}, 1 \mathrm{H}), 3.83(\mathrm{~s}, 1 \mathrm{H})$, $3.42 \sim 2.96(\mathrm{~m}, 3 \mathrm{H}), 2.46(\mathrm{~s}, 3 \mathrm{H}), 2.35 \sim 2.09(\mathrm{~m}, 2 \mathrm{H})$, $1.85(\mathrm{~s}, 2 \mathrm{H}) ;{ }^{13} \mathrm{C}$ NMR (126 MHz, Chloroform- $d$ ) $\delta$ : $170.46,165.15,150.17,147.62,145.11,137.59,131.81$ $(2 \times \mathrm{C}), 131.62,130.90,130.82,130.57(2 \times \mathrm{C}), 129.86$, $121.89,47.58,42.27,40.19,32.69,31.86,16.01$. HRMS (ESI) calcd for $\mathrm{C}_{21} \mathrm{H}_{19} \mathrm{BrCl}_{2} \mathrm{~N}_{3} \mathrm{OS}[\mathrm{M}+\mathrm{H}]^{+}$509.9804, found 509.9797 .

(4-(5-(4-(叔丁基)苯基)-4-甲基噻唑-2-基)哌啶-1基)(5,6-二氯吡啶-3-基)甲酮(6d): 收率 63.8\%, 淡黄色固 体. m.p. $160 \sim 161{ }^{\circ} \mathrm{C} ;{ }^{1} \mathrm{H}$ NMR $(500 \mathrm{MHz}$, Chloroform- $d$ ) $\delta: 8.38(\mathrm{~d}, J=2.1 \mathrm{~Hz}, 1 \mathrm{H}), 7.89(\mathrm{~d}, J=2.1 \mathrm{~Hz}$, $1 \mathrm{H}), 7.53 \sim 7.42(\mathrm{~m}, 2 \mathrm{H}), 7.40 \sim 7.33(\mathrm{~m}, 2 \mathrm{H}), 4.75(\mathrm{~s}$, $1 \mathrm{H}), 3.84(\mathrm{~s}, 1 \mathrm{H}), 3.49 \sim 2.87(\mathrm{~m}, 3 \mathrm{H}), 2.49$ (s, 3H), 2.28 (d, $J=76.0 \mathrm{~Hz}, 2 \mathrm{H}), 1.89$ (d, $J=27.0 \mathrm{~Hz}, 2 \mathrm{H}), 1.37$ (s, $9 \mathrm{H}) ;{ }^{13} \mathrm{C}$ NMR (126 MHz, Chloroform- $d$ ) $\delta:$ 169.79, $165.17,150.85,150.17,146.91,145.14,137.61,131.69$, $131.15,130.83,128.97,128.76(2 \times \mathrm{C}), 125.59(2 \times \mathrm{C})$, 47.67, 42.20, 40.20, 34.58, 32.66, 31.98, $31.21(3 \times \mathrm{C})$, 16.04. HRMS (ESI) calcd for $\mathrm{C}_{25} \mathrm{H}_{28} \mathrm{Cl}_{2} \mathrm{~N}_{3} \mathrm{OS}[\mathrm{M}+\mathrm{H}]^{+}$ 488.1325, found 488.1324 .

(5,6-二氯吡啶-3-基)(4-(4-甲基-5-苯基噻唑-2-基)哌 啶-1-基)甲酮(6e): 收率 64.5\%, 白色固体. m.p. 146 $147{ }^{\circ} \mathrm{C}$; ${ }^{1} \mathrm{H}$ NMR $(500 \mathrm{MHz}$, Chloroform- $d$ ) $\delta: 8.38(\mathrm{~d}$, $J=2.1 \mathrm{~Hz}, 1 \mathrm{H}), 7.89(\mathrm{~d}, J=2.1 \mathrm{~Hz}, 1 \mathrm{H}), 7.47 \sim 7.33(\mathrm{~m}$, $5 \mathrm{H}), 4.75(\mathrm{~s}, 1 \mathrm{H}), 3.84(\mathrm{~s}, 1 \mathrm{H}), 3.40 \sim 2.92(\mathrm{~m}, 3 \mathrm{H}), 2.49$ (s, 3H), 2.26 (s, 2H), 1.90 (s, 2H); ${ }^{13} \mathrm{C}$ NMR (126 MHz, Chloroform-d) $\delta: 170.16,165.15,150.15,147.15,145.11$, $137.58,131.89,131.65,131.09,130.80,129.07(2 \times \mathrm{C})$, $128.62(2 \times \mathrm{C}), 127.73,47.55,42.17,40.15,32.63,31.91$,
15.97. HRMS (ESI) calcd for $\mathrm{C}_{21} \mathrm{H}_{20} \mathrm{Cl}_{2} \mathrm{~N}_{3} \mathrm{OS}[\mathrm{M}+\mathrm{H}]^{+}$ 432.0699, found 432.0696.

(5,6-二氯吡啶-3-基)(4-(4-甲基-5-(3-(三氟甲基)苯 基)噻唑-2-基)哌啶-1-基)甲酮(6f)：收率 55.2\%，白色固 体. m.p. $136 \sim 137{ }^{\circ} \mathrm{C} ;{ }^{1} \mathrm{H}$ NMR $(500 \mathrm{MHz}$, Chloroform- $d) \delta: 8.38(\mathrm{~d}, J=2.1 \mathrm{~Hz}, 1 \mathrm{H}), 7.89(\mathrm{~d}, J=2.1 \mathrm{~Hz}$, $1 \mathrm{H}), 7.67$ (t, $J=1.8 \mathrm{~Hz}, 1 \mathrm{H}), 7.65 \sim 7.54(\mathrm{~m}, 3 \mathrm{H}), 4.75$ (s, 1H), 3.85 (s, 1H), 3.40 2.92 (m, 3H), 2.49 (s, 3H), 2.25 (s, 2H), 1.88 (s, 2H); ${ }^{13} \mathrm{C}$ NMR (126 MHz, Chloroform- $d$ ) $\delta: 171.01,165.23,150.25,148.21,145.14,137.62,132.89$, 132.32, 131.64, 130.88, 131.19 (q, $J=32.5 \mathrm{~Hz}), 129.52$, 129.24, 125.81 (q, $J=3.8 \mathrm{~Hz}), 124.47$ (q, $J=3.7 \mathrm{~Hz})$, 123.78 (q, $J=272.5 \mathrm{~Hz}$ ), 47.51, 42.21, 40.23, 32.69, 31.87, 15.97. HRMS (ESI) calcd for $\mathrm{C}_{22} \mathrm{H}_{19} \mathrm{Cl}_{2} \mathrm{~F}_{3} \mathrm{~N}_{3} \mathrm{OS}[\mathrm{M}+\mathrm{H}]^{+}$ 500.0572 , found 500.0568 .

(4-(5-(3-氯苯基)-4-甲基噻唑-2-基)哌啶-1-基)(5,6二氯吡啶-3-基)甲酮 $(\mathbf{6 g})$ : 收率 55.7\%, 白色固体. m.p. 156 $157{ }^{\circ} \mathrm{C} ;{ }^{1} \mathrm{H}$ NMR (500 MHz, Chloroform- $d$ ) $\delta: 8.38$ (d, $J=2.1 \mathrm{~Hz}, 1 \mathrm{H}), 7.89(\mathrm{~d}, J=2.1 \mathrm{~Hz}, 1 \mathrm{H}), 7.43 \sim 7.28$ (m, 4H), $4.74(\mathrm{~s}, 1 \mathrm{H}), 3.84(\mathrm{~s}, 1 \mathrm{H}), 3.39 \sim 2.94(\mathrm{~m}, 3 \mathrm{H})$, 2.48 (s, 3H), 2.24 (d, $J=33.8 \mathrm{~Hz}, 2 \mathrm{H}), 1.90$ (ddd, $J=20.8$, 15.6, 9.4 Hz, 2H); ${ }^{13} \mathrm{C}$ NMR (126 MHz, Chloroform- $d$ ) $\delta$ : $170.70,165.17,150.17,147.91,145.11,137.58,134.49$, $133.72,131.62,130.82,129.87,129.56,129.00,127.81$, 127.22, 47.61, 42.25, 40.16, 32.65, 31.90, 16.02. HRMS (ESI) calcd for $\mathrm{C}_{21} \mathrm{H}_{19} \mathrm{Cl}_{3} \mathrm{~N}_{3} \mathrm{OS}[\mathrm{M}+\mathrm{H}]^{+} 466.0309$, found 466.0306 .

(4-(5-(3-溴苯基)-4-甲基噻唑-2-基)哌啶-1-基)(5,6二氯吡啶-3-基)甲酮(6h): 收率 $60.3 \%$, 白色固体. m.p. $154 \sim 155{ }^{\circ} \mathrm{C} ;{ }^{1} \mathrm{H}$ NMR (500 MHz, Chloroform- $d$ ) $\delta: 8.37$ (d, $J=2.1 \mathrm{~Hz}, 1 \mathrm{H}), 7.89$ (d, $J=2.1 \mathrm{~Hz}, 1 \mathrm{H}), 7.57$ (t, $J=1.8$ $\mathrm{Hz}, 1 \mathrm{H}), 7.49$ (dt, $J=7.9,1.6 \mathrm{~Hz}, 1 \mathrm{H}), 7.38 \sim 7.26(\mathrm{~m}$, 2H), 4.74 (s, 1H), 3.84 (s, 1H), 3.40 2.97 (m, 3H), 2.47 (s, 3H), 2.35 2.10 (m, 2H), $1.87(\mathrm{~s}, 2 \mathrm{H}) ;{ }^{13} \mathrm{C}$ NMR $(126$ MHz, Chloroform- $d$ ) $\delta: 170.73,165.15,150.16,147.94$, $145.10,137.58,134.00,131.88,131.61,130.80,130.71$, $130.10,129.42,127.66,122.60,47.51,42.17,40.15,32.64$, 31.90, 16.00. HRMS (ESI) calcd for $\mathrm{C}_{21} \mathrm{H}_{19} \mathrm{BrCl}_{2} \mathrm{~N}_{3} \mathrm{OS}$ $[\mathrm{M}+\mathrm{H}]^{+}$509.9804, found 509.9803.

(5,6-二氯吡啶-3-基)(4-(4-甲基-5-(间甲基苯基)噻 唑-2-基)哌啶-1-基)甲酮(6i)：收率 53.2\%, 淡黄色固体. m.p. $134 \sim 135{ }^{\circ} \mathrm{C} ;{ }^{1} \mathrm{H}$ NMR $(500 \mathrm{MHz}$, Chloroform- $d$ ) $\delta$ : $8.38(\mathrm{~d}, J=2.1 \mathrm{~Hz}, 1 \mathrm{H}), 7.89$ (d, $J=2.1 \mathrm{~Hz}, 1 \mathrm{H}), 7.32$ (td, $J=7.2,1.5 \mathrm{~Hz}, 1 \mathrm{H}), 7.26 \sim 7.15(\mathrm{~m}, 3 \mathrm{H}), 4.75(\mathrm{~s}, 1 \mathrm{H})$, $3.84(\mathrm{~s}, 1 \mathrm{H}), 3.40 \sim 2.93(\mathrm{~m}, 3 \mathrm{H}), 2.49(\mathrm{~s}, 3 \mathrm{H}), 2.41(\mathrm{~d}, J=$ 
$3.1 \mathrm{~Hz}, 3 \mathrm{H}), 2.24(\mathrm{~s}, 2 \mathrm{H}), 1.85$ (s, 2H); ${ }^{13} \mathrm{C}$ NMR (126 $\mathrm{MHz}$, Chloroform- $d$ ) $\delta: 170.22,165.24,150.24,146.98$, $145.16,138.42$, 137.64, 131.75, 131.67, 131.35, 130.88, 129.85, 128.64, 128.58, 126.20, 47.65, 42.28, 40.17, 32.77, 31.88, 21.37, 15.96. HRMS (ESI) calcd for $\mathrm{C}_{22} \mathrm{H}_{22} \mathrm{Cl}_{2} \mathrm{~N}_{3}$ OS $[\mathrm{M}+\mathrm{H}]^{+}$446.0855, found 446.0853 .

(5,6-二氯吡啶-3-基)(4-(5-(2-氟苯基)-4 甲基噻唑-2基)哌啶-1-基)甲酮(6j)：收率 45.6\%，白色固体. m.p. $140 \sim 141{ }^{\circ} \mathrm{C} ;{ }^{1} \mathrm{H}$ NMR (500 MHz, Chloroform-d) $\delta: 8.38$ $(\mathrm{d}, J=2.1 \mathrm{~Hz}, 1 \mathrm{H}), 7.89$ (d, $J=2.1 \mathrm{~Hz}, 1 \mathrm{H}), 7.44 \sim 7.33$ $(\mathrm{m}, 2 \mathrm{H}), 7.26 \sim 7.14(\mathrm{~m}, 2 \mathrm{H}), 4.75(\mathrm{~s}, 1 \mathrm{H}), 3.83(\mathrm{~s}, 1 \mathrm{H})$, $3.37 \sim 2.93(\mathrm{~m}, 3 \mathrm{H}), 2.38(\mathrm{~s}, 3 \mathrm{H}), 2.25(\mathrm{~d}, J=34.1 \mathrm{~Hz}$, 2H), 1.92 (s, 2H); ${ }^{13} \mathrm{C}$ NMR (126 MHz, Chloroform- $d$ ) $\delta$ : 171.60, 165.15, 159.59 (d, $J=248.8 \mathrm{~Hz}), 150.14,149.70$, 145.11, 137.57, 131.97 (d, $J=2.4 \mathrm{~Hz}), 131.65,130.80$, 130.09 (d, $J=8.1 \mathrm{~Hz}), 124.14$ (d, $J=3.6 \mathrm{~Hz}), 123.62$, $119.51(\mathrm{~d}, J=15.3 \mathrm{~Hz}), 116.03$ (d, $J=22.1 \mathrm{~Hz}), 47.62$, 42.21, 40.17, 32.67, 31.89, 15.93. HRMS (ESI) calcd for $\mathrm{C}_{21} \mathrm{H}_{19} \mathrm{Cl}_{2} \mathrm{FN}_{3} \mathrm{OS}[\mathrm{M}+\mathrm{H}]^{+}$450.0604, found 450.0607 .

(4-(5-(2,3-二氯苯基)-4-甲基噻唑-2-基)哌啶 -1基)(5,6-二氯吡啶-3-基)甲酩(6k)：收率 33.2\%, 白色固 体. m.p. 162 163 ${ }^{\circ} \mathrm{C}$; ${ }^{1} \mathrm{H}$ NMR (500 MHz, Chloroform- $d) \delta: 8.42 \sim 8.34(\mathrm{~m}, 1 \mathrm{H}), 7.92 \sim 7.84(\mathrm{~m}, 1 \mathrm{H})$, $7.59 \sim 7.48(\mathrm{~m}, 1 \mathrm{H}), 7.32 \sim 7.21(\mathrm{~m}, 2 \mathrm{H}), 4.75(\mathrm{~s}, 1 \mathrm{H})$, $3.84(\mathrm{~s}, 1 \mathrm{H}), 3.38 \sim 2.90(\mathrm{~m}, 3 \mathrm{H}), 2.27(\mathrm{~s}, 5 \mathrm{H}), 1.88(\mathrm{~s}$, $2 \mathrm{H}) ;{ }^{13} \mathrm{C}$ NMR (126 MHz, Chloroform- $d$ ) $\delta: 171.78$, $165.16,150.17,150.01,145.11,137.57,133.79,133.12$, $132.82,131.62,130.81,130.80,130.66,127.02,126.84$, 47.65, 42.20, 40.17, 32.67, 31.88, 15.71. HRMS (ESI) calcd for $\mathrm{C}_{21} \mathrm{H}_{18} \mathrm{Cl}_{4} \mathrm{~N}_{3} \mathrm{OS}[\mathrm{M}+\mathrm{H}]^{+}$499.9919, found 499.9911.

(4-(5-(2,4-二氯苯基)-4- 甲基噻唑-2-基)哌啶-1基)(5,6-二氯吡啶-3-基)甲酮(61)：收率 38.6\%，白色固体. m.p. 157 $158{ }^{\circ} \mathrm{C} ;{ }^{1} \mathrm{H}$ NMR $(500 \mathrm{MHz}$, Chloroform- $d$ ) $\delta$ : $8.38(\mathrm{~d}, J=2.1 \mathrm{~Hz}, 1 \mathrm{H}), 7.91 \sim 7.85(\mathrm{~m}, 1 \mathrm{H}), 7.53$ (d, $J=$ $1.9 \mathrm{~Hz}, 1 \mathrm{H}), 7.34 \sim 7.28(\mathrm{~m}, 2 \mathrm{H}), 4.75(\mathrm{~s}, 1 \mathrm{H}), 3.84(\mathrm{~s}$, $1 \mathrm{H}), 3.32$ (tt, $J=11.4,4.0 \mathrm{~Hz}, 2 \mathrm{H}), 3.06(\mathrm{~s}, 1 \mathrm{H}), 2.28$ (s, 5H), 1.89 (s, 2H); ${ }^{13} \mathrm{C}$ NMR (126 MHz, Chloroform- $d$ ) $\delta$ : $171.95,165.18,150.18,150.06,145.12,137.58,135.34$, $135.20,133.29$, 131.62, 130.82, 129.80, 129.13, 127.10, 125.99, 47.56, 42.24, 40.17, 32.69, 31.86, 15.69. HRMS (ESI) calcd for $\mathrm{C}_{21} \mathrm{H}_{18} \mathrm{Cl}_{4} \mathrm{~N}_{3} \mathrm{OS}[\mathrm{M}+\mathrm{H}]^{+} 499.9919$, found 499.9910 .

(4-(5-(2,5-二氯苯基)-4- 甲基噻唑-2-基)哌啶-1基)(5,6-二氯吡啶-3-基)甲酮(6m)：收率 45.9\%, 白色固
体. m.p. $158 \sim 159{ }^{\circ} \mathrm{C}$; ${ }^{1} \mathrm{H}$ NMR $(500 \mathrm{MHz}$, Chloroform- $d) \delta: 8.38(\mathrm{~d}, J=2.1 \mathrm{~Hz}, 1 \mathrm{H}), 7.89(\mathrm{~d}, J=2.1 \mathrm{~Hz}$, $1 \mathrm{H}), 7.52 \sim 7.30(\mathrm{~m}, 3 \mathrm{H}), 4.75(\mathrm{~s}, 1 \mathrm{H}), 3.85(\mathrm{~s}, 1 \mathrm{H}), 3.42 \sim$ $2.93(\mathrm{~m}, 3 \mathrm{H}), 2.30(\mathrm{~s}, 5 \mathrm{H}), 1.87(\mathrm{~s}, 2 \mathrm{H}) ;{ }^{13} \mathrm{C}$ NMR $(126$ $\mathrm{MHz}$, Chloroform-d) $\delta$ : 172.14, 165.27, 150.29,150.28, $145.15,137.64,133.00,132.50,132.36,132.18,131.63$, 131.01, 130.91, 129.92, 125.89, 47.70, 42.30, 40.22, 32.73, 31.93, 15.79. HRMS (ESI) calcd for $\mathrm{C}_{21} \mathrm{H}_{18} \mathrm{Cl}_{4} \mathrm{~N}_{3} \mathrm{OS}[\mathrm{M}+$ $\mathrm{H}]^{+}$499.9919, found 499.9917 .

(4-(4-(2,6-苯乙基)噻唑-2-基)哌啶-1-基)(5,6-二氯吡 啶-3-基)甲酮(6n)：收率 52.4\%，白色固体. m.p. 136 $137{ }^{\circ} \mathrm{C}$; ${ }^{1} \mathrm{H}$ NMR (500 MHz, Chloroform- $d$ ) $\delta: 8.37$ (d, $J=2.2 \mathrm{~Hz}, 1 \mathrm{H}), 7.88$ (d, $J=2.1 \mathrm{~Hz}, 1 \mathrm{H}), 7.35$ (d, $J=8.0$ $\mathrm{Hz}, 2 \mathrm{H}), 7.18$ (t, $J=8.0 \mathrm{~Hz}, 1 \mathrm{H}), 6.52$ (t, $J=1.4 \mathrm{~Hz}, 1 \mathrm{H})$, 4.67 (s, 1H), $4.46(\mathrm{~d}, J=1.1 \mathrm{~Hz}, 2 \mathrm{H}), 3.82$ (s, 1H), $3.44-$ $2.96(\mathrm{~m}, 3 \mathrm{H}), 2.23(\mathrm{~d}, J=33.8 \mathrm{~Hz}, 2 \mathrm{H}), 1.83(\mathrm{~s}, 2 \mathrm{H}) ;{ }^{13} \mathrm{C}$ NMR (126 MHz, Chloroform- $d$ ) $\delta: 172.73,165.23,152.71$, $150.23,145.16,143.28,137.64,136.03,134.95,131.70$, $130.88,128.51,128.31(2 \times \mathrm{C}), 112.92,47.44,42.16$, 40.00, 33.43, 32.64, 31.85. HRMS (ESI) calcd for $\mathrm{C}_{21} \mathrm{H}_{17} \mathrm{Cl}_{4} \mathrm{~N}_{3} \mathrm{OSNa}[\mathrm{M}+\mathrm{Na}]^{+}$521.9739, found 521.9737 .

\section{3 生物活性测试}

\subsection{1 实验处理方法}

靶标：小麦赤霉病菌，苹果褐斑病菌，水稻纹枯病 菌, 马铃薯晚疫病菌, 黄瓜灰霉病菌.

将融化好的 PDA 培养基冷却至 $60 \sim 70{ }^{\circ} \mathrm{C}$, 按设计 浓度加入定量药剂, 配置成 $100 \mu \mathrm{g} / \mathrm{mL}$ 的含毒培养基, 待其充分冷却后, 接种直径为 $5 \mathrm{~mm}$ 的病菌菌饼, 然后 放置于培养箱中培养, $96 \mathrm{~h}$ 后，采用十字交叉法进行结 果调查。

\subsection{2 药效计算}

$$
\text { 抑菌率 }(\%)=\frac{\text { 对照菌落直径－处理菌落直径 }}{\text { 对照菌落直径一菌饼直径 }} \times 100 \%
$$

辅助材料(Supporting Information) 目标化合物 $\mathbf{6 a} \sim$ 6n 的 ${ }^{1} \mathrm{H}$ NMR, ${ }^{13} \mathrm{C}$ NMR 和 HRMS. 这些材料可以免费 从本刊网站(http://sioc-journal.cn/)上下载.

\section{References}

[1] Huang, X. F.; Tang, J. F.; Ji, J. L. J. Organomet. Chem. 2012, 878,

[2] Chavva, K.; Pillalamarri, S.; Banda, V. Bioorg. Med. Chem. Lett, 2014, 23, 5893.

[3] Dev, G. J.; Poornachandra, Y.; Reddy, K. R.; Kumar, R. N. Eur. J. Med. Chem. 2017, 130, 223

[4] Liu, R. B.; Zheng, Y. Q.; Wang, Y. L. Agrochemicals 2018, 57, 326 (in Chinese). (刘瑞宾, 郑怡倩, 汪炎鲁, 农药, 2018, 57, 326.)

[5] Cederbaum, F. WO 2014118142, 2014 [Chem. Abstr. 2014, 161 
320959].

[6] Lamberth, C. WO 2014154530, 2014 [Chem. Abstr. 2014, 161, 640429].

[7] Tomoki, T. US 20130296272, 2013 [Chem. Abstr. 2014, 156, 337332].

[8] Gregory, V. WO 2009055514, 2009 [Chem. Abstr. 2009, 150, 578441].

[9] Stefan, H. US 20160198713, 2016 [Chem. Abstr. 2016, 162, 400022].

[10] Pierre, C. US 20110312999, 2011 [Chem. Abstr. 2011, 155, 615369].

[11] Hanagan, M. A. WO 2009094407, 2009 [Chem. Abstr. 2009, 151, 234956].

[12] Kamireddy, B. WO 2009094445, 2009 [Chem. Abstr. 2009, 151, 173451].

[13] Pasteris, R. J. WO 2008013925, 2008 [Chem. Abstr. 2008, 160, 302215].

[14] Hu, D. J.; Liu, S. F.; Huang, T. H. Molecules 2009, 14, 1288.

[15] Chen, L.; Zhu, Y. J.; Fan, Z. J. J. Agric. Food Chem. 2017, 65, 745.

[16] Sulzermosse, S.; Cederbaum, F.; Lamberth, C. Bioorg. Med. Chem. 2015, 23, 2129.

[17] Choi, W. S.; Nam, S. W.; Kim, I. D.; Kim, S. H.; Park, K. H.; Bae, I. K. J. Chem. 2015, 241793.

[18] Li, F. Y.; Zhu, Y. J.; Fan, Z. J.; Xu, J. H.; Guo X. F.; Zong, G. N.;
Song, Y. Q.; Qian, X. L.; Ma, L. Y.; Wang, J. R. Chin. J. Struct. Chem. 2015, 34, 5659.

[19] Wang, K. B.; Pu, T.; Luo, Y. J. Yunnan Agric. Univ. 2018, 33, 218 (in Chinese). (王凯博, 普特, 罗艳, 云南农业大学学报, 2018, 33, 218.)

[20] Ye, X. World Pestic. 2018, 40, 63 (in Chinese). (叶萱。世界农药， 2018, 40, 63.)

[21] Xu, J.; Ma, L.; Liu, X. B. Chin. J. Org. Chem. 2018, 38, 1680 (in Chinese). (徐姣，马玲，刘秀波，有机化学, 2018, 38, 1680.)

[22] Sun, N. B.; Shen, Z. H.; Zhai, Z. W. Chin. J. Org. Chem. 2017, 37, 2044 (in Chinese). (孙娜波, 沈钟华, 翟志文, 有机化学, 2017, 37, 2044.)

[23] Dai, H.; Li, H.; Jin, Z. C. Chin. J. Org. Chem. 2016, 36, 185 (in Chinese). (戴红, 李宏, 金智超, 有机化学, 2016, 36, 185.)

[24] Dai, H.; Chen, J.; Cao, X. F. Chin. J. Org. Chem. 2016, 36, 377 (in Chinese).

(戴红, 陈佳, 曹雄飞, 有机化学, 2016, 36, 377.)

[25] Li, Q. M.; Pang, K. S.; Zhao, J. P. Chin. J. Org. Chem. 2017, 37 , 1009 (in Chinese). (李倩梅, 庞凯胜, 赵建平, 有机化学, 2017, 37, 1009.)

[26] Li, L.; Chen, H.; Lin, Y. Synth. Commun. 2007, 37, 85.

[27] Groß, A.; Schneiders, N.; Daniel, K. Tetrahedron 2008, 64, 10882. 